Meta

Journal des traducteurs

Translators' Journal

\title{
Index français des mots et des sujets traités
}

Volume 44, numéro 4, décembre 1999

URI : https://id.erudit.org/iderudit/003209ar

DOI : https://doi.org/10.7202/003209ar

Aller au sommaire du numéro

Éditeur(s)

Les Presses de l'Université de Montréal

ISSN

0026-0452 (imprimé)

1492-1421 (numérique)

Découvrir la revue

Citer ce document

(1999). Index français des mots et des sujets traités. Meta, 44(4), 661-663.

https://doi.org/10.7202/003209ar d'utilisation que vous pouvez consulter en ligne.

https://apropos.erudit.org/fr/usagers/politique-dutilisation/ 


\section{Index français des mots et des sujets traités}

Abréviations, problème des, $\mathrm{n}^{\circ}$ 2, p. 234.

Actant, $\mathrm{n}^{\circ}$ 4, p. 651.

Activités des institutions de traducteurs chinois, $\mathrm{n}^{\circ} 1$, p. 211.

Actualisation, $\mathrm{n}^{\circ} 4$, p. 529.

Adaptation, $\mathrm{n}^{\circ}$ 4, p. 657.

Adjectivation, problème de l', nº 2, p. 230.

Allitération, $\mathrm{n}^{\circ} 3$, p. 455.

Allusions péjoratives, $\mathrm{n}^{\circ} 2$, p. 315 .

Anacoluthe, $\mathrm{n}^{\circ}$ 2, p. 231.

Analogon, $\mathrm{n}^{\circ}$ 3, p. 449.

Anglais pidgin, $\mathrm{n}^{\circ} 3$, p. 519.

Animaux, voix des, $\mathrm{n}^{\circ}$ 4, p. 586.

Antia, Bassey: La traduction en anglais de la littérature francophone: perception $d u$ phénomène au Nigéria, $\mathrm{n}^{\circ} 3$, p. 517.

Anti-orienté, argument, no 3, p. 413.

Arabe-hébreu, transposition, $\mathrm{n}^{\circ}$ 3, p. 464.

Aslanov, Cyril: Les voix plurielles de la traduction de Camus en hébreu, $\mathrm{n}^{\circ} 3$, p. 448.

Aspect, $\mathrm{n}^{\circ} 4$, p. 539.

Asynchrones, arguments, no 3, p. 424.

Ballon-zéro, no 4, p. 596.

Bande dessinée, $\mathrm{n}^{\circ}$ 4, p. 583.

Belles infidèles, $n^{\circ} 3$, p. 451 .

Bible, termes tirés de la, $n^{\circ} 2$, p. 312 .

Blanco, Xavier et Pierre-André Buvet: À propos de la traduction automatique des déterminants de l'espagnol et du français, $\mathrm{n}^{\circ} 4$, p. 525.

Bruits, $\mathrm{n}^{\circ} 4$, p. 585 .

Camus, Albert, n 3, p. 448.

Choix en traduction, $\mathrm{n}^{\circ}$ 4, p. 541.

Clas, André: Hommage à Jean-Paul Vinay, $\mathrm{n}^{\circ} 2$, p. 215.

Classe d'objets, n ${ }^{\circ}$ 4, p. 532 et 575 .

Collèges de traducteurs littéraires, $\mathrm{n}^{\circ} 3$, p. 522 .

Commentaire, $n^{\circ} 2$, p. 292.

Communauté, $\mathrm{n}^{\circ} 3$, p. 489.

Complexe organisme-milieu, $\mathrm{n}^{\circ} 3$, p. 491.

Concept, no 4 , p. 575 et 579.

Concept archétypique, $\mathrm{n}^{\circ} 4$, p. 575 .

Connecteurs, $n^{\circ} 3$, p. 415.

Contexte situationnel, $\mathrm{n}^{\mathrm{o}} 1$, p. 149.

Contexte verbal élargi, $\mathrm{n}^{\circ} 1, \mathrm{p} .147$.

Contexte verbal immédiat, $\mathrm{n}^{\circ} 1$, p. 145.

Contresens, $n^{\circ} 3$, p. 451.

Co-orienté, argument, $\mathrm{n}^{\mathrm{o}} 3$, p. 413.

Coordonnées, ${ }^{\circ} 4$, p. 536.

Créations au second degré, no 2, p. 316.
Critères d'évaluation d'une traduction, $\mathrm{n}^{\circ}$ 1, p. 55 .

Décalage de styles, $\mathrm{n}^{\circ} 3$, p. 451.

Décodage, $n^{\circ} 2$, p. 218.

Démarche documentaire pour la traduction médicale, $\mathrm{n}^{\circ} 2$, p. 218.

Dénomination, $\mathrm{n}^{\circ} 4$, p. 573.

Désinences, $n^{\circ} 2$, p. 230.

Destinataire du texte d'arrivée, $\mathrm{n}^{\circ} 2$, p. 229.

Détermination, $\mathrm{n}^{\circ} 4$, p. 526.

Dictionnaires monolingues coordonnés, $\mathrm{n}^{\circ} 4$, p. 536.

Différence sémantique, $\mathrm{n}^{\circ}$ 4, p. 656.

Diki-Kidiri, Marcel: Le signifié et le concept dans la dénomination, $\mathrm{n}^{\circ} 4$, p. 572 .

Discursif, type, $\mathrm{n}^{\circ} 2$, p. 229.

Dury, Pauline: Étude comparative et diachronique des concepts ecosystem et écosystème, $\mathrm{n}^{\circ} 3$, p. 484 .

Écologie, $n^{\circ} 3$, p. 486.

Écosystème, $\mathrm{n}^{\circ} 3$, p. 485.

Écriture blanche, $\mathrm{n}^{\circ} 3$, p. 449.

Emprunt, no 4, p. 595.

Éponymie, problèmes d', $\mathrm{n}^{\circ}$ 2, p. 234.

Eurocentrisme, $n^{\circ} 4$, p. 650.

Expression des sentiments du locuteur, $\mathrm{n}^{\circ} 4$, p. 591.

Fantaisies argotiques, $\mathrm{n}^{\circ}$ 2, p. 317.

Faux amis morphologiques, $\mathrm{n}^{\circ} 2$, p. 230.

Faux comparatifs, $n^{\circ} 2$, p. 236.

Faux sens, n' 3 , p. 451.

Fidélité, $\mathrm{n}^{\circ} 1$, p. 44, 52 et 61 .

Français-espagnol, traduction, $\mathrm{n}^{\circ} 3$, p. 411.

Français-hébreu, traduction, $n^{\circ} 3$, p. 448.

Français-igbo, traduction, $n^{\circ} 4$, p. 651.

Fréquentatif, $\mathrm{n}^{\circ} 4$, p. 528 .

Histoire, termes tirés de l', n 2, p. 312.

Histoire de la traduction chinoise, $\mathrm{n}^{\circ} 1, \mathrm{p} .47$.

Hypertexte, no 2, p. 292.

Identité conceptuelle, $\mathrm{n}^{\circ} 3$, p. 489.

Image symbolique, $\mathrm{n}^{\circ} 4$, p. 575 .

Insensibilité du traducteur, $\mathrm{n}^{\circ} 3$, p. 519.

Institutions de traducteurs et d'interprètes chinois, $\mathrm{n}^{\circ} 1$, p. 210

Interférence, $\mathrm{n}^{\circ} 4$, p. 597.

Interjection, $\mathrm{n}^{\circ} 4$, p. 582 .

Interjection, méthode de traduction de l', $\mathrm{n}^{\circ} 4$, p. 593. 
Interjection, sémantisme de l', nº 4, p. 597.

Interjection, traduction de l', no 4 , p. 585 .

Interjection appellative, $\mathrm{n}^{\circ} 4$, p. 588 .

Interjection dans la bande dessinée, importance de l', no 4 , p. 584 .

Interjection expressive, $\mathrm{n}^{\circ} 4$, p. 588 .

Interjection impropre, $\mathrm{n}^{\circ} 4$, p. 589.

Interjection propre, $\mathrm{n}^{\circ} 4$, p. 587.

Interjections, classement des, $\mathrm{n}^{\circ} 4$, p. 585 .

Interjections, difficulté de traduire les, $\mathrm{n}^{\circ} 4$, p. 594.

Intraduisibilité, $\mathrm{n}^{\circ}$ 1, p. 63.

Introducteur, $\mathrm{n}^{\circ} 3$, p. 413.

Invalidation absolue, $\mathrm{n}^{\circ} 3$, p. 416.

Invalidation partielle, $\mathrm{n}^{\circ} 3$, p. 419.

Isomorphisme textuel, $\mathrm{n}^{\circ} 3$, p. 518 .

Jammal, Amal: Une méthodologie de la traduction médicale, $\mathrm{n}^{\circ} 2$, p. 217.

Jeux de mots, $\mathrm{n}^{\circ} 2$, p. 315.

Juhel, Denis: Prolixité et qualité des traductions, $\mathrm{n}^{\circ} 2$, p. 238.

Jun, Xu: Réflexions sur les études des problèmes fondamentaux de la traduction, $\mathrm{n}^{\circ} 1$, p. 44.

Juron, $\mathrm{n}^{\circ} 4$, p. 590 .

Kumarajiva, $\mathrm{n}^{\circ} 1$, p. 48.

Lexique-grammaire, ${ }^{\circ}$ 4, p. 526.

Linéarisation, $\mathrm{n}^{\circ} 4$, p. 529.

Littéralisme, $\mathrm{n}^{\circ} 3$, p. 461.

Littérature, termes tirés de la, $\mathrm{n}^{\circ} 2$, p. 312.

Littérature africaine francophone, $\mathrm{n}^{\circ} 3$, p. 517.

Littérarité, $\mathrm{n}^{\circ} 1$, p. 67.

Longueur entre TD et TA, différence de, $n^{\circ} 2$, p. 239.

Lyrisme, $\mathrm{n}^{\circ} 3$, p. 456.

MacNamee, Térence: Le rapporteur et l'interprète de conférence, $\mathrm{n}^{\circ} 2$, p. 280.

Maïeutique, $\mathrm{n}^{\circ} 2$, p. 285.

Marqueur aspectuel, $\mathrm{n}^{\circ} 4$, p. 528.

Morphème, $\mathrm{n}^{\circ}$ 4, p. 654.

Niveaux de langue, $n^{\circ} 2$, p. 229.

Niveaux de traduction, $\mathrm{n}^{\circ} 1$, p. 59.

Non orienté, argument, n 3, p. 413.

Notions, $\mathrm{n}^{\circ} 2$, p. 291.

Notions et propositions, méthode des, $\mathrm{n}^{\circ} 2$, p. 291.

Obstacles à la traduction, $\mathrm{n}^{\circ} 1, \mathrm{p} .59$.

Okiwelu, Benedict O. : La traduction des voix françaises en igbo: problèmes et solutions, $\mathrm{n}^{\circ} 4$, p. 649.

Omission, $\mathrm{n}^{\circ} 3$, p. 518.

Onomatopées, n ${ }^{\circ} 4$, p. 585 et 587.

Opérateur aspectuel, $\mathrm{n}^{\circ}$ 4, p. 532.
Ordinateur, rôle de l', nº 2, p. 287.

Organisation prototypique du sens, $\mathrm{n}^{\circ} 4$, p. 577.

Orientation interlocutoire, $\mathrm{n}^{\circ}$ 4, p. 592.

Orientation locutoire, $\mathrm{n}^{\circ} 4$, p. 589.

Percept, $n^{\circ} 4$, p. 579.

Perception culturelle, $\mathrm{n}^{\circ}$ 4, p. 577.

Perception fonctionnelle de la traduction, $\mathrm{n}^{\circ} 3$, p. 518.

Pièges du transcodage, repérage des, $\mathrm{n}^{\circ} 2, \mathrm{p} .230$. Pièges sémantiques, repérage des, $\mathrm{n}^{\circ} 2, \mathrm{p} .220$.

Prise de notes, techniques de, $\mathrm{n}^{\circ} 2$, p. 284.

Poésie française traduite en chinois, $\mathrm{n}^{\circ} 1, \mathrm{p} .181$.

Polysémie, n ${ }^{\circ} 4$, p. 577.

Prolixité, $\mathrm{n}^{\circ} 2$, p. 239.

Prolixité, causes et effets de la, no 2, p. 241.

Prolixité, nature et correctifs de la, $\mathrm{n}^{\circ} 2, \mathrm{p} .244$.

Prolixité, prévention de la, $\mathrm{n}^{\circ} 2$, p. 245.

Propositions tacites ou implicites, $\mathrm{n}^{\circ}$ 2, p. 291.

Publications des institutions de traducteurs chinois, $\mathrm{n}^{\circ} 1$, p. 212.

Quantifieur approximatif, $\mathrm{n}^{\circ} 4$, p. 540.

Quantifieur discret, $\mathrm{n}^{\circ} 4$, p. 539.

Rapporteur, n², p. 281.

Rapporteur, reformulation par le, $\mathrm{n}^{\circ} 2, \mathrm{p} .286$.

Rapporteur en temps réel, travail d'enregistrement du, $\mathrm{n}^{\circ} 2$, p. 282.

Rapporteur en temps réversible, travail analytique du, no 2 , p. 289.

Realias, conversion des, $\mathrm{n}^{\circ} 3$, p. 459.

Recréation, $n^{\circ} 1$, p. 49, 52, 61 et 66 .

Recréation, degrés de, $\mathrm{n}^{\circ} 1, \mathrm{p} .73$.

Recherche terminologique, $\mathrm{n}^{\circ}$ 2, p. 227.

Redondance, $\mathrm{n}^{\circ}$ 2, p. 242.

Réduction, $\mathrm{n}^{\circ} 1$, p. 66 .

Références culturelles, $n^{\circ} 3$, p. 459.

Référent inconnu, $\mathrm{n}^{\circ} 3$, p. 463.

Réfutation, $n^{\circ} 3$, p. 416.

Respect de l'idiolecte du personnage, $\mathrm{n}^{\circ}$ 4, p. 601.

Retraduction, $\mathrm{n}^{\circ} 1$, p. 51 .

Rétroaction, $\mathrm{n}^{\circ} 2$, p. 286.

Rey, Joëlle: Approche argumentative des textes scientifiques: la traduction de or en espagnol, $\mathrm{n}^{\circ} 3$, p. 411.

Rime interne, $\mathrm{n}^{\circ} 3$, p. 455.

Romans français traduits en chinois, $\mathrm{n}^{\circ} 1$, p. 178.

Saveur originale, transmettre la, $\mathrm{n}^{\mathrm{o}} 1$, p. 118.

Sens social connoté, rapporter le, $\mathrm{n}^{\circ} 1, \mathrm{p} .117$.

Signes des mœurs, $\mathrm{n}^{\circ} 1$, p. 116.

Signes des mœurs, interpréter, $\mathrm{n}^{\circ}$ 1, p. 119.

Signes idiomatiques, $\mathrm{n}^{\circ} 1$, p. 114.

Signes sociaux, $\mathrm{n}^{\circ} 1$, p. 111 .

Signes sociolinguistiques, $\mathrm{n}^{\circ} 1$, p. 113.

Signifiant, $n^{\circ} 4$, p. 574.

Signifié, $n^{\circ} 4$, p. 574 . 
Signifié central, $\mathrm{n}^{\circ} 4$, p. 577.

Signifié non central, n ${ }^{\circ}$, p. 577.

Situation, $\mathrm{n}^{\circ} 1$, p. 149.

Situation d'énonciation, $\mathrm{n}^{\circ} 4$, p. 598.

Sons articulés humains, $\mathrm{n}^{\circ} 4$, p. 587.

Soriano, Ascension Sierra: L'interjection dans la

BD: Réflexions sur sa traduction, $\mathrm{n}^{\circ} 4$, p. 581.

Stratification, $\mathrm{n}^{\circ} 1$, p. 56 et 58 .

Structure musicale, $\mathrm{n}^{\circ} 2$, p. 285.

Style, $n^{\circ} 1$, p. 53 et $n^{\circ} 3$, p. 450.

Synchrones, arguments, $\mathrm{n}^{\circ} 3$, p. 424.

Synonymie, problèmes de, $\mathrm{n}^{\circ} 2$, p. 233.

Système, traduction comme, $\mathrm{n}^{\circ} 1, \mathrm{p} .56$.

Temps du langage, $\mathrm{n}^{\circ} 2$, p. 287.

Textèmes, $\mathrm{n}^{\circ}$ 2, p. 291.

Textes, étude des, $\mathrm{n}^{\circ} 2$, p. 289.

Textualité, $\mathrm{n}^{\circ} 3$, p. 518.

Toponymie indigène, influence de la, $\mathrm{n}^{\mathrm{o}} 2$, p. 314 .

Traduction, enseignement de la, $n^{\circ} 4$, p. 650 .

Traduction automatique, $\mathrm{n}^{\circ} 4$, p. 525 .

Traduction exotique, $\mathrm{n}^{\circ} 1$, p. 53 .

Traduction libre, $\mathrm{n}^{\circ} 1$, p. 47.

Traduction littéraire, $\mathrm{n}^{\circ} 1$, p. 61.

Traduction médicale, $\mathrm{n}^{\circ} 2$, p. 216.

Traduction sino-centrique, $\mathrm{n}^{\circ} 1$, p. 53 .

Transcodage, $\mathrm{n}^{\circ} 2$, p. 229.

Transposition, $\mathrm{n}^{\circ} 4$, p. 657.

Transposition culturelle, $\mathrm{n}^{\circ} 3$, p. 462.
Valeur argumentative, $\mathrm{n}^{\circ} 3$, p. 412.

Validation absolue, $\mathrm{n}^{\circ} 3$, p. 426.

Van Hoof, Henri: Les noms de pays, de peuples et de lieux dans le langage imagé, $\mathrm{n}^{\circ} 2$, p. 312.

Verbe support, $\mathrm{n}^{\circ} 4$, p. 530.

Voix active, $\mathrm{n}^{\circ} 4$, p. 651.

Voix attributive, ${ }^{\circ} 4$, p. 651.

Voix moyenne, $n^{\circ} 4$, p. 652.

Voix passive, $\mathrm{n}^{\circ} 4$, p. 651.

Wei, Chen : Contexte, compréhension, traduction, $\mathrm{n}^{\circ} 1$, p. 144.

Wuilmart, Françoise: Collège européen des traducteurs littéraires de Seneffe, $\mathrm{n}^{\circ} 3$, p. 522.

Xiaoyi, Yuan: Débat du siècle: fidélité ou recréation, $\mathrm{n}^{\circ} 1$, p. 61

Xiebin, She: La littérature française traduite en Chine, $\mathrm{n}^{\circ}$ 1, p. 178.

Xiebin, She: Les institutions de traducteurs et d'interprètes chinois, leurs activités et leurs publications, $\mathrm{n}^{\circ} 1$, p. 209.

Xinmu, Zhang: Les signes sociaux et leur traduction, $\mathrm{n}^{\circ} 1$, p. 110.

Yiddish, nº 3, p. 454.

Zeugme, no 2, p. 235. 